\title{
Capillary condensation between disks in two dimensions
}

\section{Gil, Tamir; Ipsen, John Hjorth}

\section{Published in:}

Physical Review E. Statistical, Nonlinear, and Soft Matter Physics

Link to article, DOI:

10.1103/PhysRevE.55.1713

Publication date:

1997

\section{Document Version}

Publisher's PDF, also known as Version of record

Link back to DTU Orbit

Citation (APA):

Gil, T., \& Ipsen, J. H. (1997). Capillary condensation between disks in two dimensions. Physical Review E. Statistical, Nonlinear, and Soft Matter Physics, 55(2), 1713-1721. https://doi.org/10.1103/PhysRevE.55.1713

\section{General rights}

Copyright and moral rights for the publications made accessible in the public portal are retained by the authors and/or other copyright owners and it is a condition of accessing publications that users recognise and abide by the legal requirements associated with these rights.

- Users may download and print one copy of any publication from the public portal for the purpose of private study or research.

- You may not further distribute the material or use it for any profit-making activity or commercial gain

- You may freely distribute the URL identifying the publication in the public portal

If you believe that this document breaches copyright please contact us providing details, and we will remove access to the work immediately and investigate your claim. 


\title{
Capillary condensation between disks in two dimensions
}

\author{
Tamir Gil and John H. Ipsen \\ Department of Chemistry, The Technical University of Denmark, DK-2800 Lyngby, Denmark
}

(Received 26 January 1996; revised manuscript received 27 August 1996)

\begin{abstract}
Capillary condensation between two two-dimensional wetted circular substrates (disks) is studied by an effective free energy description of the wetting interface. The interfacial free-energy potential is developed on the basis of the theory for the wetting of a single disk, where interfacial capillary fluctuations play a dominant role. A simple approximative analytical expression of the interfacial free energy is developed and is validated numerically. The capillary condensation is characterized by the analysis of the coverage of the condensed phase, its stability, and asymptotic behaviors. The theory can be applied to the description of flocculations in two-dimensional systems of colloids. [S1063-651X(97)05802-9]

PACS number(s): 68.45.Gd, 87.22.Bt, 64.70.Fx
\end{abstract}

\section{INTRODUCTION}

The close relationship between wettability of surfaces, nucleation, and condensation phenomena is of great importance in many natural and technological processes. The formation of a liquid phase from a gaseous phase between solid surfaces, so-called capillary condensation, provides a classical example. Recently, it has been pointed out on the basis of experimental observations that wetting of the surfaces in a colloidal suspension may be the cause of flocculation due to capillary condensation [1]. In this work we investigate the conditions for this effect in a two-dimensional system.

Capillary condensation in spherical geometry has been considered theoretically by use of mean-field theory of an effective interface description of the configuration of the wetting film around spheres [2] and around lines and lattices of cylinders [3]. In the present paper we extend this work to the two-dimensional analogous system of two disks and concentrate on some additional aspects, e.g., the wetting-induced aggregation of the disks. Further, our study is motivated by the rich properties of biological membranes. Biomembranes are quasi-two-dimensional systems that display in-plane phase transitions and phase separations under natural conditions [4]. Therefore, wetting effects may appear in the presence of large inclusions, such as large integral proteins (or rather complexes of them). The biophysical significance of our results will be discussed elsewhere [5].

There is a crucial difference between wetting phenomena in two and three spatial dimensions that is due to the renormalizing effect of the capillary-wave fluctuations of the interface between the wetting phase and the thermodynamically stable phase in the background. Because three is the marginal dimension for this renormalizing effect [6], the relative importance of these fluctuations for the thermodynamics of wetting phenomena in three dimensions is expected to be small compared with the direct forces involved, e.g., van der Waals forces. In two dimensions though (onedimensional interface), the capillary-wave fluctuations play a dominant role giving rise to an effective long-range repulsive force between the substrate and the fluid-fluid interface and exclude the possibility of prewetting transitions [6,7]. As a result, once direct substrate-interface interactions are not strong enough (above the wetting temperature) to hold the interface close enough to the surface of the substrate, the mean thickness of the wetting film is determined by the balance between a disjoining pressure, induced by the capillary fluctuations and tending to enlarge the mean thickness, and external pressures that tend to diminish it, e.g., hydrostatic pressure or gravity. Such a phenomenological picture was recently shown to apply to the wetting of a large circular substrate (disk) if a Laplace pressure is added to the pressure balance in the wetting film [8]. The mean thickness of the wetting film then grows like $r_{0}^{1 / 3}$, for $r_{0} \rightarrow \infty$, where $r_{0}$ is the radius of the substrate [8] (see Sec. II). Hence a macroscopic wetting film of thickness much larger than the molecular distances emerges at large values of $r_{0}$, validating the use of interface models in which the density profile of the fluidfluid interface is replaced by a sharp kink to which a local interfacial stiffness is attached [6]. In this paper we expand the effective interface potential of the single disk to the case of two disks by adding a fluctuation-induced potential (Sec. II) to the interface potential of Ref. [2] (Sec. IV). In this way we include the dominating effect of the long-wavelength fluctuations though remaining in the simple mean-field framework of Ref. [2].

Under the conditions that trigger wetting of a single disk, bringing two disks close to each other, gives rise to two expected topologies of the fluid-fluid interface line between the wetting phase and the thermodynamically stable phase in the background: one of two separate loops, closing around each one of the disks individually [Fig. 1 (sep)], and one of a single loop wrapping the two disks [Fig. 1 (bri)]. The latter is due to capillary condensation between the two disks. A transition between the separated and bridged configurations can be induced either by tuning the distance between the disks or by changing the thermodynamic conditions for the system, e.g., the chemical potential difference between the wetting phase and the background phase. This transition can be characterized, for example, by the discontinuous jump in the coverage of the wetting phase or in the aggregation force, both jumps occurring as capillary condensation takes place. The aggregation force is caused by the tendency of the condensed system to reduce the length of the interface and the coverage of the thermodynamically unstable wetting phase by reducing the distance between the disks and is of course vanishing when the disks separate. In Sec. V, we 


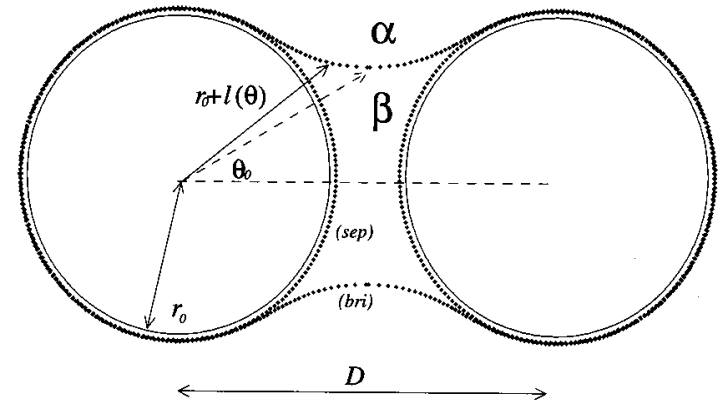

FIG. 1. Two two-dimensional disks of radius $r_{0}$ lying at a distance $D$. The wetting films surrounding each one of the disks (sep) remain separated until $\Pi_{b}$ is sufficiently small to allow the formation of a bridging interface (bri). The equilibrium $\alpha$ - $\beta$ interface line is described by $r_{0}+l(\theta)$, where $\theta$ is measured from the line connecting the centers of the two disks and $\theta_{0}$ is the angular position of the center of the bridge.

discuss those features and illustrate them graphically in addition to calculating the relations among the thermodynamic parameters at the points where the capillary condensation occurs.

Even though the problem of capillary condensation between two disks is very much simplified by the use of an effective interfacial potential, the equilibrium form of this potential, which we write in Sec. IV, can only be obtained numerically. However, an analytical approximation of this potential can be reached by splitting the system into two systems that effect each other only through the boundary conditions: one system describing the fluid-fluid interface far from the substrate, in the region between the two disks, and the other system describing the fluid-fluid interface in the regions where it wraps the rest of the disks. In Sec. III, the effect of capillary-wave fluctuations and the boundary conditions on the analytical approximation are discussed and asymptotic properties are derived. In Sec. V we compare the effective interfacial potential of Sec. IV with the analytical approximation of Sec. III. We start our discussion by rederiving a fluctuation-induced effective potential for the single disk.

\section{FLUCTUATION-INDUCED EFFECTIVE POTENTIAL IN THE CASE OF A SINGLE DISK}

In this section we derive a fluctuation-induced effective potential per unit radial angle of a circular substrate from the theory of wetting of a single disk [8]. We shall apply this effective potential in the description of capillary condensation between two disks in Secs. III and IV below.

Consider a fluid system close to a first-order phase transition line between two fluid phases $\alpha$ and $\beta$. Roughly speaking, fluctuations in such a system can be described by what we shall call the bulk correlation length and define as

$$
\xi_{b} \equiv k_{B} T / \sigma
$$

where $\sigma$ is the stiffness (energy per unit length) of the $\alpha-\beta$ interface, $k_{B}$ is the Boltzmann constant, and $T$ is the temperature [9]. Hence one can refer to bulk domains ( $\alpha$ or $\beta$ ) in this system only if their typical size $r$ is much larger than the bulk correlation length, i.e.,

$$
r \gg \xi_{b} .
$$

When condition (2) is fulfilled, one can describe capillary fluctuations of a two-dimensional $(d=2) \alpha-\beta$ interface of an underlying circular geometry by the phenomenological capillary Hamiltonian (see, for example, Ref. [8])

$$
\mathcal{H}[r(\theta)]=\mathcal{H}_{0}[r(\theta)]+\int_{0}^{2 \pi} W[r(\theta)] d \theta,
$$

where

$$
\mathcal{H}_{0}[r(\theta)]=\int_{0}^{2 \pi} d \theta\left[\sigma r(\theta)+\frac{\sigma}{2 r(\theta)}\left(\frac{d r}{d \theta}\right)^{2}\right]
$$

describes the fluctuations of the "free" interface and $W(r)$ is some $r$-dependent effective potential of local character (does not include derivatives of $r$ ) that accounts for all possible constraints put on the $\alpha-\beta$ interface. $r(\theta)$ measures the local distance of the interface from the origin at a given angular position $\theta$. The partition function of such a system $\mathcal{Z}$ is calculated by

$$
\mathcal{Z}=\int \mathcal{D} \operatorname{rexp}\left\{-\mathcal{H}[r(\theta)] / k_{B} T\right\}
$$

where $\mathcal{D r}$ is the functional measure for the integration over all possible $r(\theta)$ functions satisfying the periodic boundary condition

$$
r(0)=r(2 \pi)
$$

As discussed in Ref. [8], when $W \equiv 0$ and the $\alpha-\beta$ system is governed only by the stiffness of the $\alpha-\beta$ interface and thermal fluctuations, the size $r$ of the $\beta$-like domains produced by fluctuations does not satisfy condition (2). However, the situation is different if a " $\beta$-preferring" circular solid substrate is present in the system. Let us denote by $\gamma$ such a two-dimensional disk-shaped substrate of radius $r_{0}$ and suppose that the energy of creating a $\beta-\gamma$ interface is much lower than the one involved in creating an $\alpha-\gamma$ one. Above a certain temperature (the wetting temperature), the preferred phase $\beta$ tends to form a layer intruding between the substrate and the other phase $\alpha$, even when the latter is stable in the bulk. When the thickness of this $\beta$ like wetting layer is much larger than the width of its interfaces with the $\alpha$ and $\gamma$ phases, we say that the $\beta$-phase wets the disk. The width of those interfaces is comparable with the sum of the bulk correlation lengths involved. When the substrate is a solid, the width of the interfaces is solely determined by the correlation lengths in the $\alpha$ and the $\beta$ phases.

Denoting the thickness of the wetting film by $l$, one can apply the interface Hamiltonian approach [6] only if [see Eq. (2)]

$l \gg \xi_{b}$. 
When condition (7) is fulfilled, capillary fluctuations of the $\alpha-\beta$ interface are described by the capillary Hamiltonian (3) with the periodic boundary conditions (6) and with $W$ given by $[8]$

$$
W[r(\theta)]=\frac{1}{2} \Pi_{b}\left[r^{2}(\theta)-r_{0}^{2}\right]+d u(r, \theta),
$$

where $\Pi_{b}$ is a pressurelike field that measures the difference in the grand canonical potentials per unit area between the $\alpha$ and $\beta$ phases (at coexistence, $\Pi_{b}=0$ ) so that the first in term Eq. (8) is proportional to the area of the wetting film accounting for the bulk contribution coming from adsorbing a thermodynamically unfavorable phase. $u(r)$ is an effective substrate-interface interaction potential that accounts for the corrections to $\mathcal{H}$ coming from direct molecular interactions and it contains the crucial hard-core part that excludes the $\alpha-\beta$ interface from the substrate area.

The mean thickness of the wetting film $l_{W}$ has recently been determined by applying transfer-matrix techniques to calculate the partition function (5) with the boundary conditions (6) [8]. Criterion (7) was shown to be satisfied only in what we shall define as the complete wetting regime

$$
r_{0} \gg \xi_{b}, T_{W}<T<T_{C}, \sigma / r_{0} \gg \Pi_{b} \rightarrow 0,
$$

where $T_{C}$ is the bulk $\alpha$ - $\beta$ critical point and $T_{W}$ is the wetting temperature for the analogous planar system. In this regime $l_{W}$ is given exactly by

$$
l_{W}=(3 \sigma)^{-1 / 3}\left(k_{B} T\right)^{2 / 3} z_{0}^{2 / 3} \Pi_{\mathrm{eff}}^{-1 / 3} \simeq 1.238 \sigma^{-1 / 3}\left(k_{B} T\right)^{2 / 3} \Pi_{\mathrm{eff}}^{-1 / 3},
$$

where $z_{0}$ is the zeroth solution for the equation for the $J$ Bessel functions

$$
J_{1 / 3}\left(z_{n}\right)=-J_{-1 / 3}\left(z_{n}\right)
$$

and

$$
\Pi_{\mathrm{eff}} \equiv \Pi_{b}+\sigma / r_{0} .
$$

It should be noted that in this study, the lower limit of $\xi_{b}$ is $k_{B} T_{W} / \sigma$. In models for wetting in two dimensions $T_{W}$ can be very low [10] and $\xi_{b}$ can in principle go all the way down to the molecular size.

Integrating the equation of state (10), it is possible to write an effective interface grand canonical potential $\Omega(l)$, which has a minimum at $l=l_{W}$,

$$
\Omega(l)=2 \pi r_{0} \frac{C\left(k_{B} T\right)^{2}}{\sigma l^{2}}+2 \pi \sigma\left(r_{0}+l\right)+\pi \Pi_{b}\left[\left(r_{0}+l\right)^{2}-r_{0}^{2}\right] .
$$

The term $\pi \Pi_{b}\left[\left(r_{0}+l_{W}\right)^{2}-r_{0}^{2}\right]$ accounts for the excess energy of the thermodynamically unfavorable $\beta$ phase that covers an area of $\pi\left[\left(r_{0}+l_{W}\right)^{2}-r_{0}^{2}\right], 2 \pi \sigma\left(r_{0}+l\right)$ is the selfenergy of the interface, and the first term represents the loss of configurational entropy involved in preventing the interface from crossing the surface of the substrate [8]. $C \simeq 0.948$ is a universal constant that does not depend on the details of the molecular interactions in the system. Conse- quently, we define a fluctuation-induced effective potential per unit radial angle of a circular substrate as

$$
V(l) \equiv 0.948 \frac{r_{0}\left(k_{B} T\right)^{2}}{\sigma l^{2}} .
$$

$V(l)$ is of longer range than the relevant van der Waals substrate-interface interaction potential that is proportional to $r_{0} / l^{p-3}$ in the limit $l \ll r_{0}$, where $p=6,7$ for nonretarded and retarded interactions, respectively [8] and is therefore the only relevant interaction potential in the problem [7]. Thus potential (12) has a very general applicability for circular substrate-interface problems in two dimensions.

\section{CAPILLARY CONDENSATION BETWEEN TWO DISKS: SIMPLE CONSIDERATIONS}

Imagine now a system consisting of two disks of the type described in Sec. II embedded in the $\alpha$ - $\beta$ two-dimensional matrix and found in the complete wetting conditions (9) for the single disk (Fig. 1). At coexistence, $\Pi_{b} \rightarrow 0^{+}$, we can roughly say that the excess free energy of the single disk is proportional to the length of the $\alpha-\beta$ interface surrounding it, which is equal to $2 \pi\left(r_{0}+l_{W}\right) \approx 2 \pi r_{0}$ [note that from Eq. (10) $\left.l_{W} \propto r_{0}^{1 / 3} \ll r_{0}\right]$. At distances $D$ between the two disks for which

$$
D<D_{C} \approx \pi r_{0} \approx 3 r_{0},
$$

the total length of an interface wrapping the two disks together, approximately equal to $2\left(D+\pi r_{0}\right)$ is shorter than the sum of the two interfaces around two separated disks, approximately equal to $2\left(2 \pi r_{0}\right)$, making the creation of a straight bridging interface (capillary condensation) energetically favorable.

Departing from the $\alpha-\beta$ coexistence, $\Pi_{b}>0$, the bridging interface curves so that it excludes the maximum $\beta$ coverage per a given length of interface (Fig. 1). To get an idea of the shape of this "bridging" interface, we fix its two (four) ends at two (four) symmetric points, mirror to the position $(\phi, \bar{r})$, where $\phi$ is the angular position of the meeting point between the wetting interface arcs, centered on the disks, and the bridging interface line, connecting the two wetting interfaces. $\bar{r} \equiv r_{0}+\bar{l}$ is the radius of the wetting arc, where $\bar{l}$ is the mean thickness of the wetting film [11] (Fig. 2). If we ignore for a while the direct effect of the substrates apart from setting the boundary conditions and the effect of the capillarywave fluctuations, we get that the mean location of the bridging interface $\breve{y}$ is lying on a circle of radius

$$
\breve{R}=\sigma / \Pi_{b},
$$

which is determined by the Laplace pressure $\sigma / \breve{R}$ that compensates for the $\Pi_{b}$ pressure.

We can make a rough estimate of the effect of fluctuations of the $\alpha-\beta$ bridging interface for small values of the field $\Pi_{b}$. For that we consider Cartesian coordinates with the horizontal axis $(x)$ coinciding with the segment connecting the centers of the two disks and with the origin in the middle of this segment (Fig. 2). The end points at which the bridging interface is fixed are now denoted by $y\left(-x_{1}\right)$ and $y\left(x_{1}\right)$ and 


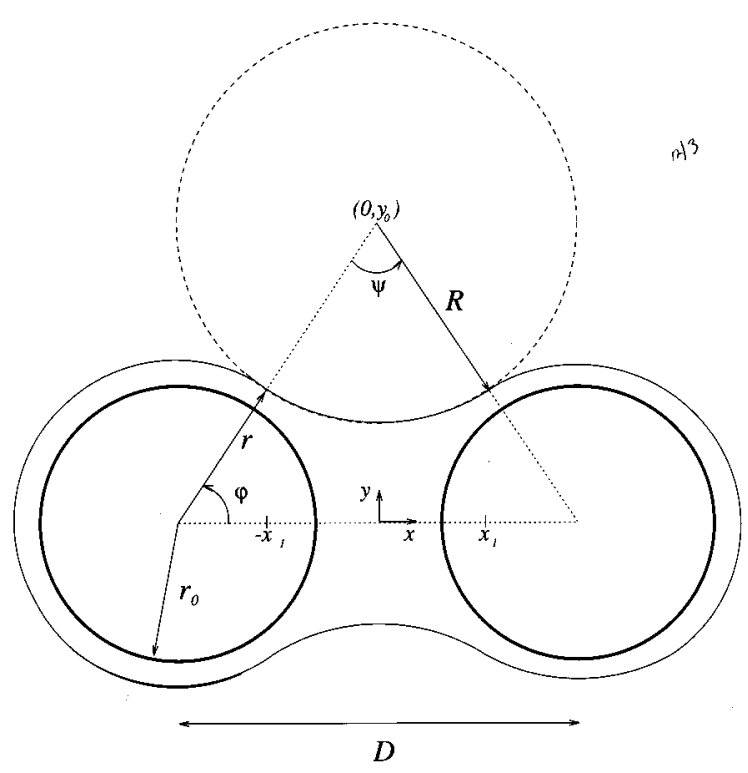

FIG. 2. Approximative construction for the equilibrium $\alpha-\beta$ interface line. The arcs of radius $r=r_{0}+l_{W}$, centered on the two disks, are connected by two tangential arcs of radius $R=\sigma / \Pi_{b}$, which are determined by the Laplace pressure $\sigma / R$. The location of the connecting points are given by $(\phi, r)$ and by $\left(x_{1}, y_{1}\right)$, and by a twofold symmetry, in polar and Cartesian coordinates, respectively.

the configurational fluctuations are assumed to be well described by the capillary-wave Hamiltonian

$$
\widetilde{\mathcal{H}}_{e}[y(x)]=\int_{-x_{1}}^{x_{1}}\left[\sigma\left(1+\frac{y_{x}^{2}}{2}\right)+\Pi_{b} y\right] d x .
$$

Fixing the end points to the values determined by the mean location $\breve{y}$, i.e., $y\left(-x_{1}\right)=y\left(x_{1}\right)=\breve{y}\left(x_{1}\right)$, the partition function of $\widetilde{\mathcal{H}}_{e}[y(x)]$ can readily be solved by standard pathintegral techniques $[12,13]$. The corresponding free energy can be expressed as

$$
\begin{aligned}
\Omega_{e} & =\widetilde{\mathcal{H}}_{e}[\widetilde{y}(x)]-\frac{k_{B} T}{2} \ln \left(\frac{\xi_{b}}{4 x_{1} \pi}\right) \\
& =k_{B} T\left\{2 \frac{x_{1}}{\xi_{b}}\left[1+\frac{\widetilde{y}\left(x_{1}\right)}{\breve{R}}-\frac{1}{6}\left(\frac{x_{1}}{\breve{R}}\right)^{2}\right]+\frac{1}{2} \ln \left(\frac{4 x_{1} \pi}{\xi_{b}}\right)\right\},
\end{aligned}
$$

where $\widetilde{y}(x)$ is the saddle-point configuration that functionally minimizes Eq. (15) and is a parabolic approximation to the circular $\breve{y}$ that lies on a circle of radius $\breve{R}$ [Eq. (14)]. $\xi_{b}=k_{B} T / \sigma$ is the bulk correlation length (1). The logarithmic contribution to the free energy is due to capillary-wave fluctuations. It is clear that in the limit of interest, i.e., $x_{1} / \xi_{b} \gg 1 \gg x_{1} / \breve{R}$, this contribution is small compared to the contribution coming from the saddle-point approximation. Furthermore, this correction to the saddle-point approximation is independent of $\Pi_{b}$, meaning that the mean position $\bar{y}(x)$ of the $\alpha-\beta$ bridging interface is not affected by thermal fluctuations. This situation is very different from the case of interfacial fluctuations close to a substrate (discussed in Sec.
II), which have a significant effect on the average position of the interface. In our model we can therefore ignore the contributions to the free energy coming from capillary fluctuations in the bridging regions while accounting for them in the wetting regions.

A naive construction of a continuous interface line for the system of two disks goes back to Ref. [14] and is illustrated in Fig. 2. The wetting interface arcs of radius $\bar{r}=r_{0}+\bar{l}$, centered on the disks, are extended to bridge between the two disks by a tangential arc of radius $\breve{R}=\sigma / \Pi_{b}$ [Eq. (14)]. The continuity is thus achieved merely by demanding

$$
\phi=\cos ^{-1}[(D / 2) /(\bar{r}+\breve{R})],
$$

where $\phi$ is the angular position of the point where the two interfaces meet each other (Fig. 2). We couple the length of the resulting interface to its effective stiffness $\sigma$ and the $\beta$ coverage to $\Pi_{b}$, and after some manipulations we obtain the following approximation for the grand canonical potential per disk of the interface wrapping the two disks:

$$
\begin{aligned}
\Omega_{1}= & \sigma[2 \vec{r}(\pi-\phi)-\breve{R}(2 \phi-\pi / 2)]+2(\pi-\phi) \widetilde{V}\left(\bar{r}-r_{0}\right) \\
& +\Pi_{b}\left[\frac{D / 2}{\breve{R}+\bar{r}} \sqrt{1-\left(\frac{D / 2}{\breve{R}+\bar{r}}\right)^{2}}\left(r_{0}^{2}+2 \breve{r} \breve{R}+\breve{R}^{2}\right)\right. \\
& \left.+\phi\left(R^{2}-\vec{r}^{2}\right)+\pi\left(\vec{r}^{2}-r_{0}^{2}\right)\right],
\end{aligned}
$$

where $\widetilde{V}\left(\bar{r}-r_{0}\right)=\widetilde{V}(\bar{l})$ is some repulsive interaction potential, e.g., the fluctuation-induced potential (12), that ensures the existence of a wetting film and that is considered here only for the region of the wetting interface of angular position between $\phi$ and $2(\pi-\phi)$. An approximative solution to the problem can now be obtained by minimizing $\Omega_{1}$ with respect to $\bar{r}$, but we will only extract some general properties. The explicit dependence on the distance between the disks only comes through the dimensionless parameter [see also Eq. (17)]

$$
\epsilon \equiv \frac{D / 2}{\bar{r}+\breve{R}} \approx \frac{D /\left(2 r_{0}\right)}{1+\frac{\sigma}{\Pi_{b} r_{0}}} \ll \frac{3}{4},
$$

considering that $D<\Pi r_{0}$ [Eq. (13)] and $\sigma /\left(\Pi_{b} r_{0}\right)>1$ [Eq. (9)]. This allows us to Taylor expand around $(D / 2) /(\bar{r}+\breve{R})=0$. The value of $\bar{r}$ that minimizes $\Omega_{1}$ obeys

$$
-\frac{\partial \widetilde{V}\left(\bar{r}-r_{0}\right)}{\partial \bar{r}}=\left(\sigma+\Pi r_{0}\right)\left[1-\frac{1}{\pi} \frac{D / 2}{\breve{R}+r_{0}}+O\left(\left(\frac{D / 2}{\breve{R}+r_{0}}\right)^{2}\right)\right] \text {. }
$$

Comparing with Eqs. (10) and (11), it is clear that the correction to the thickness of the wetting film around the disks due to the bridging is small $[O(\epsilon)]$. We will therefore proceed by assuming $\widetilde{V}(\bar{l})=V\left(l_{W}\right)$ of Eq. (12).

By insertion of Eq. (20) into Eq. (18), we find that the free energy in the bridged configuration to lowest order in $\epsilon$ has the form 


$$
\begin{aligned}
& \widetilde{\Omega}_{\mathrm{bri}} \approx f D+\sigma \pi\left(r_{0}+l_{W}\right)+\pi V\left(l_{W}\right) \\
& +\Pi_{b} \frac{\pi}{2}\left[\left(r_{0}+l_{W}\right)^{2}-2 r_{0}^{2}\right],
\end{aligned}
$$

where the coefficient $f=\sigma+\Pi_{b} r_{0}$ signifies a constant attractive force between the bridged disks up to a critical distance $\widetilde{D}_{C}=\widetilde{D}_{C}\left(T, \sigma, \Pi_{b}\right)$, at which the two disks separate. The relations between the border values of $\sigma, \Pi_{b}$, and $D$ at the transition points between the bridged and the separated configurations are approximately defined by setting $\widetilde{\Omega}_{\text {bri }}$ equal to potential (11) for the separated disk. Omitting then terms of the order of $\left(l_{W} / r_{0}\right) \ll 1$, one obtains

$$
\Pi_{b} r_{0}^{2} \approx \sigma r_{0}\left(\frac{\pi-\widetilde{D}_{C} / r_{0}}{\widetilde{D}_{C} / r_{0}-\pi / 2}\right)+\left(\frac{1}{\widetilde{D}_{C} /\left(\pi r_{0}\right)-1 / 2}\right) V\left(l_{W}\right) .
$$

Hence, for a given $D$, the values of $\sigma$ and $\Pi_{b}$ at the transition points relate to each other in an almost linear way.

It is noted that the discussion from Eqs. (20)-(22) only applies to the limit of small $\Pi_{b}$, where the properties of the system can be expressed in terms of the wetting behavior of the separated disks. For larger values of $\Pi_{b}$, the equilibrium description of the bridging disks becomes more complex. Description (18) involves the main assumption that the fluctuation-induced wall potential $V$ is only important in the wetting region $\pi-\phi \geqslant \theta \geqslant \phi$, ignoring its continuously decaying effect on the bridging interface. This does not allow a free interplay among all leading effects in determining the actual shape of the interface line. Rather it leads to the unnatural discontinuity in the curvature of the $\alpha-\beta$ interface in the meeting points between the wetting and bridging interfaces. In the following (Sec. V) we will evaluate approximation (18) by comparison with numerical solutions for the interface potential and see that it gives rise to small quantitative errors.

\section{EFFECTIVE INTERFACE POTENTIAL FOR THE CASE OF TWO DISKS}

Sections II and III have taught us that the main contribution of interfacial fluctuations to the free energy of our system comes from their exclusion by an unpenetrable substrate and is proportional to $1 / l^{2}$, where $l$ is their separation from the substrate [15]. In approximation (18) this effect is limited to the wetting films around the disks. However, in this section we relax this limitation and include the contribution of the interfacial fluctuations in an effective interface potential to describe capillary condensation between two disks.

The case of two separated wetting films is properly described by the phenomenological potential (11), which produces the correct equation of state (10) by minimization with respect to $l$. However, in the case of the bridged configuration, the rotational symmetry of the interface configurations is broken, and it is necessary to include nonhomogeneous ( $\theta$-dependent) configurations in the effective potential describing the system. In the spirit of the Landau theory, we make the natural extension of potential (11) to nonhomogeneous configurations, which vary at lengths much larger than the interfacial correlation length $\xi_{\|}[8]$, and write an effective grand canonical potential per disk for the case of two disks in the complete wetting regime (9) [16]:

$$
\begin{aligned}
\Omega[l(\theta)]= & 2 \int_{\theta_{0}}^{\pi} d \theta \sigma \sqrt{\left(r_{0}+l\right)^{2}+l_{\theta}^{2}}+2 \int_{\theta_{0}}^{\pi} d \theta V(l) \\
& +\Pi_{b}\left\{2\left[\int_{\theta_{0}}^{\pi} d \theta \frac{\left(r_{0}+l\right)^{2}}{2}\right]+\frac{D^{2}}{4} \tan \theta_{0}-\pi r_{0}^{2}\right\},
\end{aligned}
$$

where a symmetry between the two disks and a $l(\theta)=l(2 \pi-\theta)$ symmetry for each disk have been assumed. $l_{\theta} \equiv \partial l / \partial \theta$ and $\theta_{0}$ is the angular position of the center of the bridge (Fig. 1). $V(l)$ is given by Eq. (12). From left to right, the first term in $\Omega[l(\theta)]$ is the excess energy of the interface proportional to its length. The second term is the entropy term, accounting for the effect of the interfacial fluctuations in the vicinity of an unpenetrable substrate, and the third term is the excess energy of having a thermodynamically unfavorable bulk phase and is proportional to the coverage of the $\beta$ phase [see also Eq. (11)]. The only explicit temperature dependence in $\Omega$ comes from the $V(l)$ term. $\sigma$ is also temperature dependent, but it can be considered constant as long as the temperature does not enter the scaling regime for $\sigma$, i.e., is not too close to $T_{C}$, consistent with conditions (2) and (9), which require that one not get too close to $T_{C}$.

The separated and bridged cases differ from each other in the boundary conditions (see Fig. 1),

$$
l_{\theta}\left(\theta_{0}\right)=\left\{\begin{array}{lll}
0, & \theta_{0}=0 & (\text { separated disks }) \\
-D /\left(2 \sin \theta_{0}\right), & \theta_{0}>0 & (\text { bridged disks })
\end{array}\right.
$$

and

$$
l_{\theta}(\pi)=0 \quad \text { for both cases. }
$$

The interface line $l(\theta)$ is the one minimizing potential (23) under these boundary conditions, i.e., the solution to the Euler-Lagrange equation

$$
\frac{d}{d \theta}\left(\frac{\partial \Omega}{\partial l_{\theta}}\right)-\frac{\partial \Omega}{\partial l}=0
$$

Substituting $\Omega$ [Eq. (23)] into Eq. (26) results in the nonlinear differential equation

$$
\begin{aligned}
0= & l_{\theta \theta}-\left(r_{0}+l\right)\left[1+\frac{2 l_{\theta}^{2}}{\left(r_{0}+l\right)^{2}}\right] \\
& -\frac{1}{\sigma}\left[\left(r_{0}+l\right)^{2}+l_{\theta}^{2}\right]^{3 / 2}\left[\frac{h}{\left(r_{0}+l\right)}+\frac{r_{0}(\partial V / \partial l)}{\left(r_{0}+l\right)^{2}}\right] .
\end{aligned}
$$

Equation (27) can only be solved numerically and it is thus hard to analyze and understand the physics associated with description (23). However, a comparison between the numerical solutions and approximation (18) will provide some understanding of the leading physical effects (see Secs. $\mathrm{V}$ and $\mathrm{VI}$ ). 


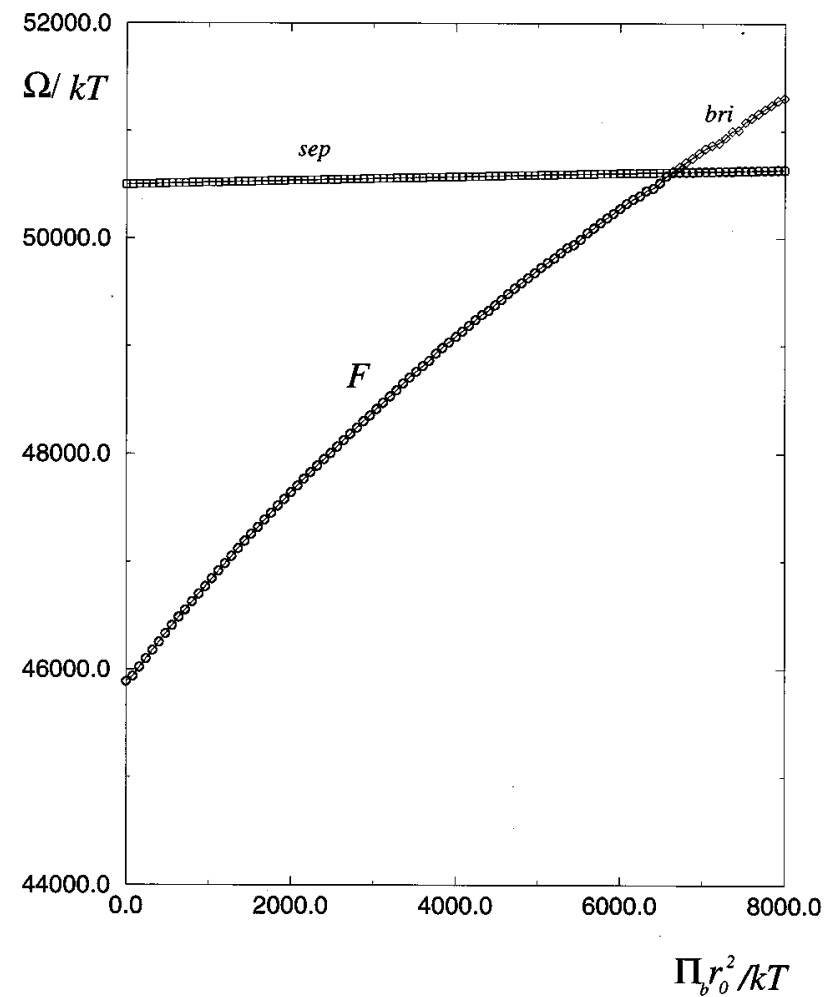

FIG. 3. Grand canonical potentials for the bridged (diamonds) and the separated (squares) configurations are plotted against the scaled field $\Pi_{b} r_{0}^{2} / k_{B} T$ for $r_{0}=1000, \quad D=2.5 r_{0}$, and $\sigma / k_{B} T=8000 / r_{0}$. The free-energy-like function $\mathcal{F}=\Omega_{\text {bri }}$ $-k_{B} T \ln \left[1+\left(\Omega_{\mathrm{bri}}-\Omega_{\mathrm{sep}}\right) / k_{B} T\right]$ is represented by circles and clearly follows the minimal value of the grand canonical potential.

\section{COVERAGE OF THE WETTING PHASE, AGGREGATION FORCE, AND PHASE BOUNDARIES OF THE CONDENSED SYSTEM}

In Secs. III and IV we have written a phenomenological approximation [Eq. (18)] and a more rigorous effective potential [Eq. (23)], respectively, describing the capillary condensation between two disks. We will now determine some characteristic features of the capillary condensation and compare the two descriptions quantitatively in order to be able to evaluate approximation (18).

We have solved Eq. (27) numerically using the relaxation method for the two-value boundary problem [17]. Numerical values of potential (23) for the bridged and the separated configurations $\Omega_{\text {bri }}$ and $\Omega_{\text {sep }}$, respectively, are plotted in Fig. 3 versus the pressure field $\Pi_{b}$. We relate a free-energy-like function $\mathcal{F}$ to those potentials by considering them to be the only relevant (coarse-grained) configurations and by weighing them with the corresponding Boltzmann factor

$$
\mathcal{F}=-k_{B} T \ln \left[\exp \left\{-\Omega_{\text {bri }} / k_{B} T\right\}+\exp \left\{-\Omega_{\text {sep }} / k_{B} T\right\}\right] .
$$

The behavior of $\mathcal{F}$ at the point where the graphs of $\Omega_{\text {bri }}\left(\Pi_{b}\right)$ and $\Omega_{\text {sep }}\left(\Pi_{b}\right)$ meet (Fig. 3) indicates a first-order phase transition between the two configurations in the thermodynamic limit. However, since we are not dealing with a thermodynamic limit and there is always a finite probability

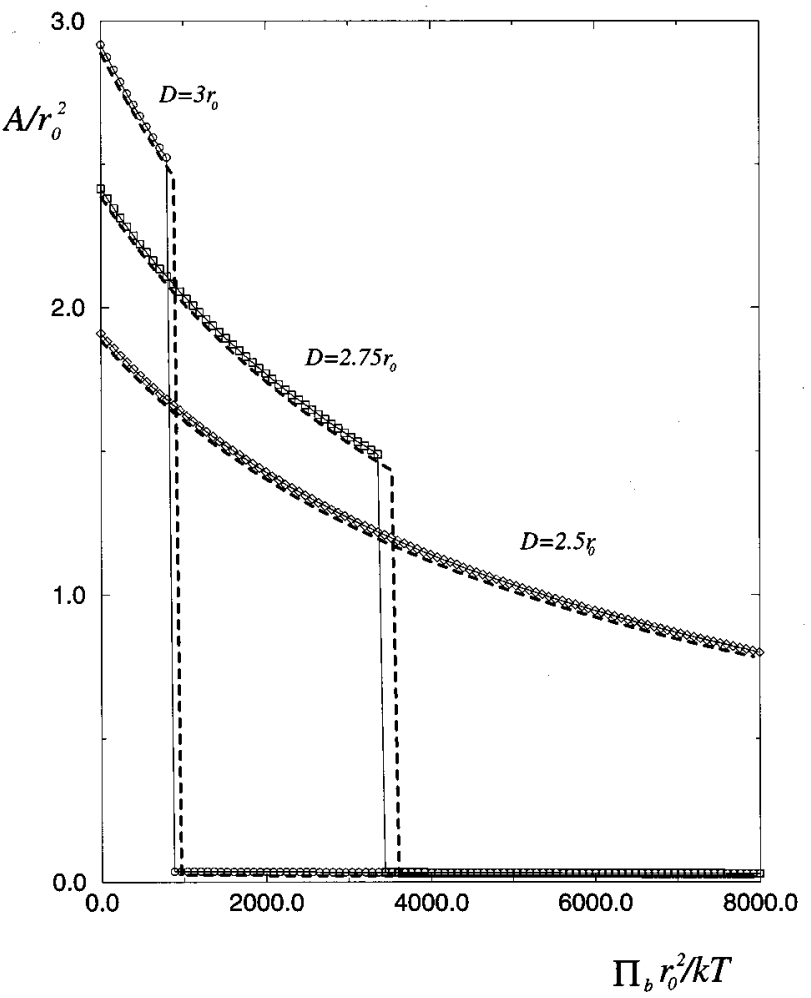

FIG. 4. Scaled coverage of the $\beta$ phase $A / r_{0}^{2}$ plotted against the scaled field $\Pi_{b} r_{0}^{2} / k_{B} T . r_{0}=1000$ and $\sigma / k_{B} T=8000 / r_{0}$. Three different values of separation $D$ between the disks are considered: $D / r_{0}=3.0$ (circles), 2.75 (squares), and 2.5 (diamonds), where it is observed that at $D=2.5 r_{0}$ the disks remain bridged within the complete wetting regime. The dashed lines are derived from approximation (18) for the grand canonical potential. The values of $\Pi_{b} r_{0}^{2} / k_{B} T$ above which the wetting films are separated, and where $A / r_{0}^{2}$ goes effectively to zero, fall with the growth of $D$.

for switching from the one configuration to the other, we cannot really relate thermodynamic phases to the bridged or to the separated configurations, and the term "phase transition" remains as a borrowed concept appealing only to the intuition. To differ from the three-dimensional case of two spheres [2], no thin-bridge-thick-bridge transition is observed. Such a transition is not to be expected in the lower dimensionality of two.

The features of the transition between the bridged and the separated configurations are nicely illustrated by two observables, the coverage $A$ of the $\beta$-like wetting phase and an aggregation force $F$ that pulls the disks together once a bridged configuration is created. These two observables exhibit a jump at the transition point. The coverage is naturally given as a function of the pressure field $\Pi_{b}$ (Fig. 4), while the aggregation force is given as a function of the distance $D$ between the disks (Fig. 5).

The $\beta$ coverage is defined formally by $A=\partial \mathcal{F} / \partial \prod_{b}[$ see Eq. (28)]. In Fig. 4 it is calculated in two ways: In the first way Eq. (27) is solved numerically and $A$ is calculated by "Boltzmann averaging" on $A_{\text {bri }}$ and $A_{\text {sep }}$ for the bridged and separated configurations, respectively. In the second way $A$ is calculated by $\partial \Omega_{1} / \partial \Pi_{b}$ derived from approximation (18). The two resulting graphs lie very close to each other. However, the values of $\partial \Omega_{1} / \partial \Pi_{b}$ are slightly lower, leading to a 


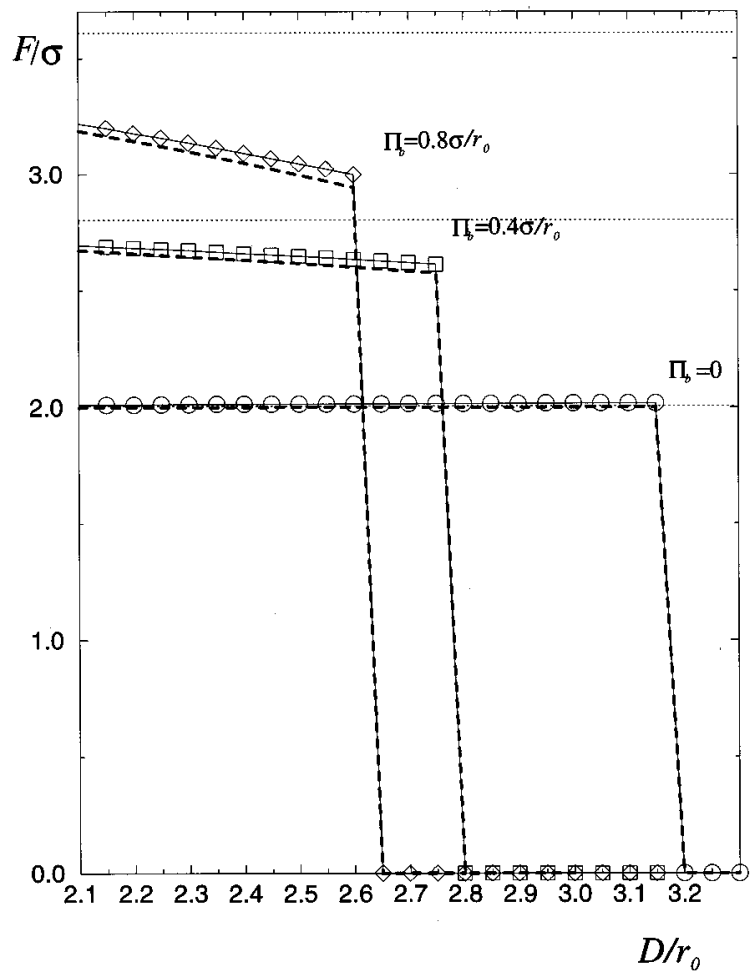

FIG. 5. Scaled aggregation force $F / \sigma$ versus the distance $D / r_{0} \cdot r_{0}=1000, \sigma / k_{B} T=3000 / r_{0}$, and $\Pi_{b} r_{0} / \sigma=0$ (circles), 0.4 (squares), and 0.8 (diamonds). The dashed lines are derived from approximation (18). The dotted lines are the values of $2 f / \sigma=2\left(1+\Pi_{b} r_{0} / \sigma\right)$ [see Eq. (21)], valid at the limit of $\Pi_{b} \rightarrow 0$. The value of $F / \sigma$ increases with $\Pi_{b}$, while its range decreases.

separation at higher values of $\Pi_{b}$.

The aggregation force $F$ is given by $F \equiv-2 \partial \mathcal{F} / \partial D$ [18], where $\mathcal{F}$ is the free energy (28). When plotting $F$ against $D$ with a zero $\Pi_{b}$ field, we observe that the disks separate at $D / r_{0} \approx 3.15$ (Fig. 5) as expected from estimate (13) for $D_{C}$. The corresponding $\beta$ coverage in Fig. 4, namely, $A\left(\Pi_{b} \rightarrow 0, D / r_{0}=3\right)$, agrees with the simple estimate of $A_{C} / r_{0}^{2} \approx 2 D_{C} / r_{0}-\pi \approx 3$. As $\Pi_{b} \rightarrow 0, \Omega_{\text {bri }}$ is given by Eq. (21) so that $F \equiv-2 \partial \mathcal{F} / \partial D$ is given by $2 f=2\left(\sigma+\Pi_{b} r_{0}\right)$. In Fig. 5 we indeed see that $F\left(\Pi_{b}=0\right)=2 \sigma$. For values of $\Pi_{b}$ larger than zero, $F$ falls almost linearly with the distance $D$, exhibiting a negative slope of a size increasing with $\Pi_{b}$. One can clearly see how the actual values of $F$ depart from the asymptotic value of $F=2 f$ as $\Pi_{b}$ is increased. But $F_{1} \equiv-2 d \Omega_{1} / d D$, derived from approximation (18), fits very well to the numerical solutions of Eq. (27). A particularly compact form for $F$ is obtained by taking the limit $r=r_{0}$. This changes the curves of $F_{1}=d \Omega_{1}(r) / d D$ in a negligible way, but yields

$$
\frac{2}{\sigma} \frac{d \Omega_{1}\left(r=r_{0}\right)}{d D}=2 \frac{1+h\left(1-\frac{\left(D / 2 r_{0}\right)^{2}}{1+1 / h}\right)}{\sqrt{1-\left(\frac{D / 2 r_{0}}{1+1 / h}\right)^{2}}},
$$

where $h \equiv \Pi_{b} r_{0} / \sigma$ is a dimensionless field.

Following the position of the transition between the bridged and the separated configurations in the $\left(\sigma r_{0}, \Pi_{b} r_{0}^{2}\right)$

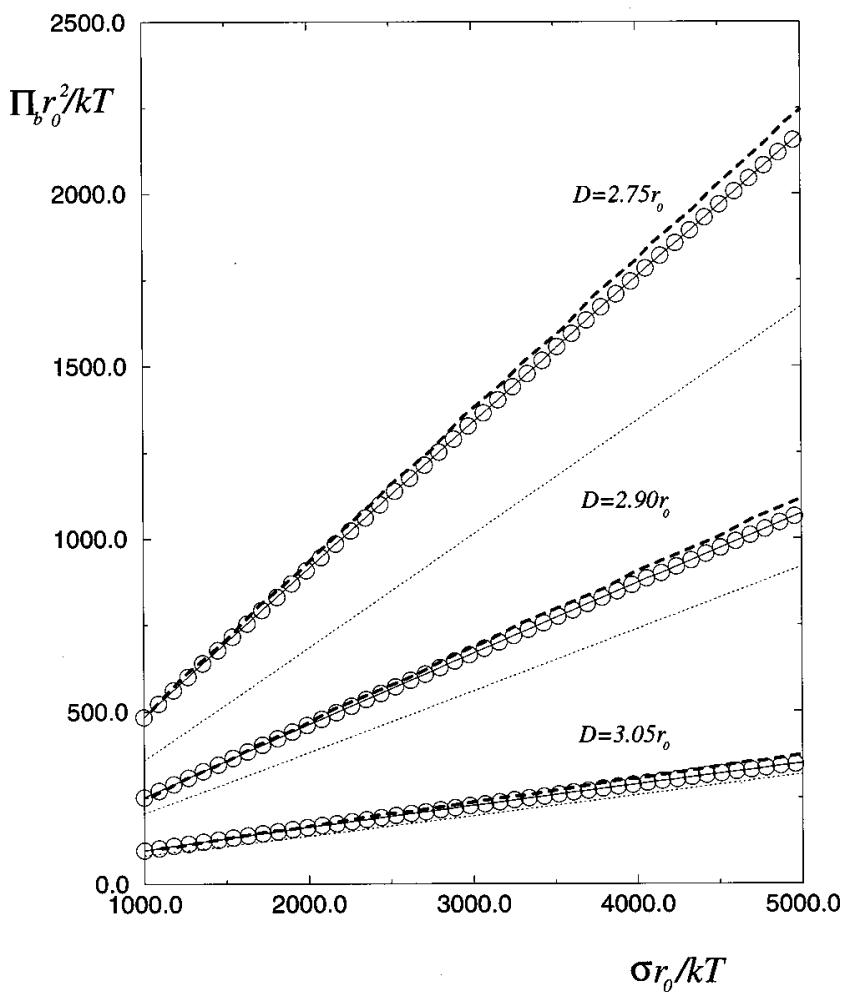

FIG. 6. Values of the scaled field $\Pi_{b} r_{0}^{2} / k_{B} T$ against the scaled stiffness of the interface $\sigma r_{0} / k_{B} T$ at the transition between the bridged and the separate configurations for three different values of the distance between the disks, $D / r_{0}=2.75,2.90$, and 3.05, where $r_{0}=1000$. The dashed lines are calculated from approximation (18). The dotted lines are given by approximation (22), valid at the limit of $\Pi_{b} \rightarrow 0$.

space, we observe an approximately linear behavior (Fig. 6). For very low values of the field $\Pi_{b}$, this behavior was analyzed in Eq. (22). At large values of $\Pi_{b}$, Eq. (22) deviates from the graphs coming from the numerical solutions of Eq. (27) and approximation (18). Nevertheless, Eqs. (27) and (18) agree with each other very well, maintaining the almost linear behavior (Fig. 6).

The two examples above, Figs. 5 and 6, have demonstrated that there is a region of positive values of $\Pi_{b}$, close to zero, in which approximation (21) is applicable. In all cases (Figs. 4-6), approximation (18) agrees very well with the numerical solutions of Eq. (27).

\section{CONCLUSION}

In conclusion, we have studied capillary condensation between two disks in two dimensions as an extension of a theory recently advanced for the wetting of a single disk. Our model has not been restricted to any specific system and it can be applied to any two-dimensional problem that involves wetting of disks. Moreover, our approach can easily be extended to describe aggregation phenomena among more than two "wetted" disklike objects in two dimensions. Such phenomena may be observable in lipid membranes with large but finite inclusions and near a first-order phase transition (or phase separation) in the membrane.

We have rigorously shown that in two dimensions, where 
capillary-wave fluctuations play a dominant role, capillary condensation between two disks can be described within a simple mean-field framework similar to that used to describe capillary condensation between two spheres in three dimensions [2] [see Eq. (23)]. This is made possible by the proper inclusion of the disjoining pressure, which stabilizes the wetting films around the disks against chemical and hydrostatic pressures that tend to diminish it. In two dimensions, this disjoining pressure is dominated by the fluctuations induced effective potential (12), which is exact and universal and overrules the effects coming from van der Waals interaction potentials. Hence, unlike the disjoining pressure in three dimensions, it does not depend on the molecular details nor does it rely on any a priori assumption concerning the nature of the molecular interactions, e.g., the additive interaction energy between the volume elements (see Refs. [19] and [2]). This fundamental difference between the two- and the three-dimensional systems, together with the difference of geometry, which is reflected, for example, in the fact that prewettinglike transitions are found in three dimensions, but not in two $[20,8]$, gives rise to different wetting behaviors.

The effective interfacial free energy for the wetting interface close to a circular substrate above the wetting temperature is the basis for the description of the capillary condensation between two disks. Capillary condensation between two wet disks occurs already when the distance between the substrates is of order of their radius $r_{0}$. It involves a dramatic increase in the local concentration (or rather the coverage) of the wetting phase (see Fig. 4) and introduces a new effective force in the system, giving rise to a net attraction between the disks (see Fig. 5). The detailed description of this phenomenon can be obtained by the use of analytical approximation schemes or a numerical approach to obtain the interface configuration, which minimizes the interfacial free energy $[2,3]$. The analytical approximation (18) is based on a simple construction (see Fig. 2) in which the differentiability of the interface configuration is relaxed in the small regions where the interface is detaching from the single disk to bridge between the two disks. We have systematically shown [Eq. (16)] that the effect of capillary-wave fluctuations is negligible in the regions where the interface is bridging between the two disks and, by an expansion around a small parameter [Eq. (20)], to a leading order the thickness of the remaining wetting film is given by the theory for the separated disk. To complete the picture, we have shown by comparison (Sec. V) that the simple analytical approximation (18) of the equilibrium interface potential gives a very good description of the condensation phenomena that is more rigorously defined by potential (23).

The advantage of approximation (18) lies in the ability to evaluate the relative weights of the different contributions to the interface potential and to systematically calculate asymptotic behaviors for limit values of the different parameters. For example, very close to the phase transition $\Pi_{b} \rightarrow 0$, the aggregation of the disks can be expressed in terms of the wetting of the single disk and the attractive force between the aggregated disks is approximatively constant and of size $F=2\left(\sigma+\Pi_{b} r_{0}\right)$ [see Eq. (21)]. Further, Eq. (18) adds only three unknowns-the fluid-fluid interfacial tension, the distance between the disks and their radii-to the laboratory controlled fields (temperature and pressure). This gives it a predictive power and makes it suitable in the analysis of experimental results.

\section{ACKNOWLEDGMENTS}

We are grateful to O. G. Mouritsen for following and promoting this study as well as for a critical reading of the manuscript and very valuable comments. This work has been supported by the Danish Natural Science Research Council under Grant No. 9400091.
[1] D. Beysens and D. Esteve, Phys. Rev. Lett. 54, 2123 (1985).

[2] H. T. Dobbs, G. A. Darbellay, and J. M. Yeomans, Europhys. Lett. 18, 493 (1992).

[3] W. R. Osborn and J. M. Yeomans, Phys. Rev. E 51, 2053 (1995)

[4] M. K. Jain, in Membrane Fluidity in Biology, edited by R. C. Aloia (Academic, New York, 1983), Vol. 1.

[5] M. Sabra, T. Gil, J. H. Ipsen, and O. G. Mouritsen (unpublished)

[6] Reviews of wetting phenomena can be found for example in S. Dietrich, in Phase Transition and Critical Phenomena, edited by C. Domb and J. L. Lebowitz (Academic, New York, 1988), Vol. 12; M. Schick, in Liquids at Interfaces, 1988 Les Houches Lectures, edited by J. Charvolin, J. F. Joanny, and J. Zinn-Justin (North-Holland, Amsterdam, 1990).

[7] R. Lipowsky and M. E. Fisher, Phys. Rev. B 36, 2126 (1987).

[8] T. Gil and L. V. Mikheev, Phys. Rev. E 52, 772 (1995).

[9] Close to the bulk $\alpha$ - $\beta$ critical point $T_{C}$, where the bulk correlation length is assumed to be the same in the $\alpha$ and in the $\beta$ phases, this definition coincides with the hyperscaling relation $\xi_{b}^{d-1}=$ const $\times k_{B} T / \sigma$. However, the theory here is rel- evant also for temperatures much lower than $T_{C}$.

[10] This situation is typical for models for wetting in two dimensions where the fluid-fluid interface is always rough and the system is found in the strong fluctuation regime as analyzed and defined in Ref. [7].

[11] We write $\bar{l}$ and not $l_{W}$ in order not to exclude the possibility that the value of $\bar{l}$, minimizing the potential, may be different from $l_{W}$ [Eq. (10)], although we later on show that to the relevant order of approximation $\bar{l}=l_{W}$.

[12] R. P. Feynman and A. R. Hibbs, Quantum Mechanics and Path Integrals (McGraw-Hill, New York, 1965).

[13] L. S. Schulman, Techniques and Applications of Path Integration (Wiley, New York, 1981).

[14] H. M. Princen, J. Colloid Interface Sci. 30, 69 (1969).

[15] The effect of the interface-interface interaction between the upper and the lower bridging interfaces should not be ignored automatically. However, the average distance between those interfaces never goes below the order of $r_{0}$, making such effects negligible.

[16] This potential is of the same form as the analogous one in Ref. [2]. However, the fluctuation term replaces the van der Waals- 
like interaction potential term and the term coupled to the chemical potential $\Pi_{b}$ is modified due to the different geometry.

[17] W. H. Press, B. P. Flannery, S. A. Teukolsky, and W. T. Vetterling, Numerical Recipes (Cambridge University Press, Cambridge, 1989), p. 588.

[18] Since all potentials have been calculated per disk, forcelike observables between the two disks are given by twice the relevant derivatives.

[19] M. P. Gelfand and R. Lipowsky, Phys. Rev. B 36, 8725 (1987).

[20] G. A. Darbellay and J. M. Yeomans, J. Phys. A 23, 5655 (1990). 